\title{
ON ANALYTICITY AND PARTIAL DIFFERENTIAL EQUATIONS
}

\author{
BY FELIX E. BROWDER ${ }^{1}$
}

Communicated April 18, 1962

Introduction. It is the purpose of the present note to present in outline some results established in a forthcoming paper of the writer [2] on solutions of a family of partial differential equations depending upon a parameter. The questions treated were suggested (though somewhat obliquely) by a reading of the paper by Kodaira and Spencer [4] concerning solutions of differentiable families of elliptic differential operators on a vector bundle over a compact manifold, with applications to problems concerning variation of complex structure. The results with which we are concerned in [2] relate essentially to the drastically different case of noncompact manifolds. For the sake of simplicity in the present exposition, we shall restrict ourselves to differential operators operating on scalar functions on an open subset of Euclidean $n$-space.

Let $G$ be an open subset of the Euclidean $n$-space $E^{n}, M_{1}$ a realanalytic manifold. We consider a family $\left\{A_{t}\right\}$ of differential operators with (possibly) variable coefficients on $G$, with the coefficients depending also upon the parameter $t$ in $M_{1}$. Thus in the usual notation for partial differential operators,

$$
\begin{gathered}
\left(D_{j}=i^{-1} \partial / \partial x_{j}, \alpha=\left(\alpha_{1}, \cdots, \alpha_{n}\right), D^{\alpha}=\prod_{j=1}^{n} D_{j}^{\alpha_{j}},|\alpha|=\sum_{j=1}^{n} \alpha_{j}\right), \\
A_{t}=\sum_{|\alpha| \leqq r} a_{\alpha}(x, t) D^{\alpha} .
\end{gathered}
$$

We say that $\left\{A_{t}\right\}$ is an analytic family if each $a_{\alpha}$ is a real-analytic function on $G \times M_{1}$.

The questions we pose concerning such a family $\left\{A_{t}\right\}$ are the following:

(I) Does there exist a family of fundamental solutions $e_{t}(x, y)$ for $A_{t}$ on $G$ which depend analytically upon $t$ in $M_{1}$ and which for a given parameter value $t_{0}$ in $M_{1}$ coincide with a prescribed fundamental solution $e_{0}(x, y)$ for $A_{t_{0}}$ ?

(II) If $f$ is an analytic function from $M_{1}$ into the analytic functions on $G$, does there exist a family of solutions $\left\{u_{t}\right\}$ of the equations

\footnotetext{
1 The preparation of this paper was partially supported by N.S.F. Grant G-19751.
} 


$$
A_{t} u_{t}=f_{t},
$$

$t \in M_{1}$,

on $G$ with $u_{t}$ depending analytically upon $t$ in $M_{1}$ and such that for a given parameter value $t_{0}$ in $M_{1}, u_{t_{0}}$ coincides with a prescribed solution $u_{0}$ of $A_{t_{0}}\left(u_{0}\right)=f_{t_{0}}$.

(III) If $G_{1}$ is a given pre-compact open subset of $G$ and if $\left\{v_{t}\right\}$ is an analytic family of solutions of the equations $A_{t} v_{t}=0$ on $G$, can the $v_{t}$ be approximated in various function-space topologies on $G_{1}$ by solutions $u_{t}$ of $A_{t} u_{t}=0$ on $G$ with the family $\left\{u_{t}\right\}$ also analytic in $t$ on $M_{1}$ ?

These questions are a generalization of questions posed for the case of a single elliptic operator (with no parameters) and affirmatively resolved in the final section of Malgrange's Thesis [6]. We describe below some general classes of differential operators, elliptic and nonelliptic, for which positive answers may be given to some or all of the above questions.

An important feature of the results stated below, which we should emphasize, is that the manifold $M_{1}$ of the parameter values is prescribed in advance and the analytic families of solutions and fundamental solutions which we construct are analytic in the large, i.e., upon the whole of $M_{1}$ and not merely upon some suitably small neighborhood of a given point of $M_{1}{ }^{2}$

1. Let $C_{c}^{\infty}(G)$ be the family of infinitely differentiable functions with compact support in $G$, and let $L^{2}(G)$ be the usual Hilbert space formed from the complex-valued functions square-integrable with respect to Lebesgue $n$-measure on $G$. We denote the norm in $L^{2}(G)$ by $\|\cdot\|$.

For each $t$ in $M_{1}$, let $A_{t}^{\prime}$ be the formal adjoint operator of $A_{t}$, i.e.,

$$
A_{t}^{\prime} u=\sum_{|\alpha| \leq r} D^{\alpha}\left(\bar{a}_{\alpha}(x, t) u\right)
$$

Definition 1. (a) The minimal operator $A_{0, t}$ of $A_{t}$ in $L^{2}(G)$ is the closure as an operator in $L^{2}(G)$ of $A_{t}$ restricted to $C_{c}^{\infty}(G)\left(t \in M_{1}\right)$. Similarly, we denote by $A_{0, t}^{\prime}$ the minimal operator of $A_{t}^{\prime}$ in $L^{2}(G)$.

(b) The maximal operator $A_{1, t}$ of $A_{t}$ in $L^{2}(G)$ is defined by $A_{1, t}$ $=\left(A_{0, t}\right)^{*}$.

Definition 2. The closed operator $T$ in $L^{2}(G)$ is said to be a solvable

2 The writer has recently received a preprint of a note by J. F. Treves summarizing results obtained by the latter on fundamental solutions and solutions of equations with constant coefficients on $E^{n}$ depending on a parameter. For constant coefficient operators of constant strength, Treves obtains fundamental solutions of convolution type analytic in the parameter but only for a neighborhood of a given parameter value. It would be interesting to know under what condition elementary solutions of convolution type exist on $E^{n}$ which are analytic in the large in the parameter. 
realization of $A_{t}$ for a given $t$ in $M_{1}$ if $A_{0, t} \subseteq T \subseteq A_{1, t}$ while $T$ maps its domain one-to-one onto $L^{2}(G)$.

DEFINITION 3. For a fixed $t$ in $M_{1}$, the locally summable function $e$ on $G \times G$ is said to be a fundamental solution of $A_{t}$ if

$$
\begin{aligned}
& \left(A_{t}^{\prime}\right)_{y} \bar{e}(x, y)=\delta_{x}, \\
& \left(A_{t}\right)_{x} e(x, y)=\delta_{y} .
\end{aligned}
$$

We shall impose some general conditions from which the existence of analytic families of solutions and fundamental solutions may be deduced. These are given in the following two definitions:

DEFINITION 4. The differential operator $A_{t}$ is said to be proper if there exists a constant $c(t)$ such that

$$
\|u\| \leqq c(t)\left\|A_{t} u\right\|
$$

for all $u$ in $C_{c}^{\infty}(G)$.

(Equivalently, $A_{t}$ is proper if the minimal operator $A_{0, t}$ is one-toone and has a closed range in $L^{2}(G)$.)

Definition 5. The family $\left\{A_{t}\right\}$ is said to be analytically uniform if there exists a fixed linear subset $W$ of $L^{2}(G)$ and a function $U$ from $M_{1}$ into the injective bounded linear transformations of $L^{2}(G)$ such that for each $t$ in $M_{1}, U_{t}$ maps $W$ onto $D\left(A_{0, t}\right)$ while for each fixed w in $W$, $A_{0, t} U_{t} w$ is a weakly analytic function from $M_{1}$ to $L^{2}(G)$.

The most important special case of Definition 5 is that in which the operators $A_{t}$ are "equally strong," i.e., the domain of the minimal operator $D\left(A_{0, t}\right)$ is independent of $t$, while for each $w$ in $D\left(A_{0, t_{0}}\right)$, $A_{t} w$ is analytic in $t$ on $M_{1}$.

Let us examine briefly some classes of differential operators for which the conditions of Definitions 4 and $\mathbf{5}$ can be enforced. We assume that $G$ is pre-compact and that there exists an open subset $G^{\prime}$ containing $\bar{G}$ such that each $a_{\alpha}$ is analytic on $G^{\prime} \times M_{1}$.

(1) Every operator with constant coefficients is proper.

(2) An elliptic operator $A_{t}$ with analytic coefficients is proper.

(3) The operator $A_{t}$ is proper if the following two conditions are satisfied:

(i) $D\left(A_{0, t}\right)$ is contained in a subset $W^{\prime}$ of $L^{2}(G)$ which is a Banach space with respect to a norm such that the imbedding map into $L^{2}(G)$ is compact.

(ii) Every solution $u$ in $L^{2}(G)$ of the equation $A_{t} u=0$ in the distribution sense on $G^{\prime}$ such that $u$ has compact support in $G^{\prime}$ must be the null solution.

For the subset $W^{\prime}$ of (i), we may take a Sobolev space $W^{i, 2}(G)$ with $j>0$. Thus, (i) and (ii) follow from known results about the domains 
of minimal operators and about uniqueness of solutions of the Cauchy problem for suitable domains $G$ and $G^{\prime}$ for hyperbolic operators (Leray [5]) and operators of principal normal type having a strongly pseudo-convex level function (Hormander [3]). ${ }^{3}$

(4) Some examples of families $\left\{A_{t}\right\}$ which are analytically uniform by having a fixed domain for the minimal operators $A_{0, t}$ are: elliptic operators of a fixed order; formally hypoelliptic operators of equal strength; constant coefficient operators of equal strength; hyperbolic operators having top-order terms independent of $t$; and operators of principal normal type having pseudo-convex level functions and with top-order terms independent of $t$.

(5) A simple example of an analytically uniform family with $U_{t}$ not the identity map is provided by the case where the top-order terms of the hyperbolic family $\left\{A_{t}\right\}$ are obtained from those of $A_{0}$ by an analytic family of coordinate transformations.

Theorem 1. Let $\left\{A_{t}\right\}$ be a family of differential operators on $G$ depending upon $t$ in $M_{1}$. Suppose that each $A_{t}$ and $A_{t}^{\prime}$ is proper and that the families $\left\{A_{t}\right\}$ and $\left\{A_{t}^{\prime}\right\}$ are analytically uniform. Then:

(a) There exists a family of closed operators $\left\{T_{t}\right\}$ for $t$ in $M_{1}$ such that each $T_{t}$ is a solvable realization for $A_{t}$ in $L^{2}(G)$ while $t \rightarrow T_{t}^{-1}$ is an analytic function from $M_{1}$ to the bounded linear operators on $L^{2}(G)$. If $t_{0}$ is a given parameter value, $T_{0}$ a prescribed solvable realization of $A_{t_{0}}$, then the family $T_{t}$ may be chosen so that $T_{t_{0}}=T_{0}$.

(b) The kernels $e_{t, x, v}$ of the mappings $T_{t}^{-1}$ (L. Schwartz [7]) are an analytic family of distribution fundamental solutions of the corresponding $A_{t}$.

(c) If $A_{t}$ is elliptic for every $t$, the map $t \rightarrow e_{t, x, y}$ yields an analytic map of $M_{1}$ into $L_{\mathrm{loo}}^{1}(G \times G)$ with $e_{t, x, y}$ analytic on $M_{1} \times[G \times G-\Delta]$, where $\Delta$ is the diagonal of $G \times G$.

(d) If $f$ is an analytic map from $M_{1}$ into $L^{2}(G)$, then $u_{t}=T_{t}^{-1} f_{t}$ gives an analytic family of solutions of the equation $A_{t} u_{t}=f_{t}$ in $L^{2}(G)$. If $A_{t}$ is elliptic for all $t$, and $f$ is analytic in $x$ and $t$, then $u_{t}(x)$ is analytic in $(t, x)$ on $M_{1} \times G$. If $u_{0}$ is a given solution of $A_{t_{0}} u_{0}=f_{0}$ in $L^{2}(G)$, the family of solvable realizations $T_{t}$ may be chosen so that $u_{t_{0}}=u_{0}$.

From the proof of Theorem 1, we extract one step which we state as an interesting result in its own right. This is the following:

TheOREM 2. Let $A_{t}$ be a family of differential operators on $G$ depending on $t$ in $M_{1}$ such that each $A_{t}^{\prime}$ is proper and the family $\left\{A_{t}^{\prime}\right\}$ is

3 Added in proof. Similar results to those of [3] have been obtained independently in a paper of A. P. Calderon which has not yet appeared. 
analytically uniform. Let $S_{t}$ be the closed subspace of $L^{2}(G)$ consisting of solutions of the homogeneous equation $A_{t} u=0$ (in the distribution sense). If $P_{t}$ is the orthogonal projection operator of $L^{2}(G)$ onto $S_{t}$, then the map $t \rightarrow P_{t}$ is an analytic mapping of $M_{1}$ into the projections of $L^{2}(G)$ with the norm topology. In particular, $P_{t}$ is continuous in $t$ in the norm topology, so that the angle between $S_{t}$ and $S_{t_{0}}$ approaches zero as $t \rightarrow t_{0}$.

If $A_{t}$ is hypoelliptic for each $t, S_{t}$ coincides with the ordinary solutions of $A_{t} u=0$ in $C^{\infty}(G) \cap L^{2}(G)$.

2. To drop or weaken the conditions of Theorem 1, which in the examples cited above is equivalent in practical terms to assuming that either $G$ is not pre-compact or that the coefficients of $A_{t}$ need not be regular up to the boundary of $G$, we restrict ourselves to the case of elliptic operators.

TheOREM 3. Let $\left\{A_{t}\right\}$ be an analytic family of elliptic differential operators of order $r$ on $G$ for $t$ ranging over $M_{1}$. Then there exists a family of bi-regular fundamental solutions $e_{t}(x, y)$ for $A_{t}$ on $G$ such that the map $t \rightarrow e_{t}$ is an analytic mapping of $M_{1}$ into $L_{\text {loc }}^{1}(G \times G)$ while $e$ is analytic on $M_{1} \times[G \times G-\Delta]$.

TheOREM 4. Let $\left\{A_{t}\right\}$ be an analytic family of elliptic differential operators of order $r$ on $G, f_{t}(x)$ analytic on $M_{1} \times G$. Then there exists $a$ family of solutions $u_{t}(x)$ of the equation $A_{t} u_{t}=f_{t}$ with $u$ analytic on $M_{1} \times G$.

THEOREM 5. Let $\left\{A_{t}\right\}$ be an analytic family of elliptic differential operators of order $r$ on $G$. Suppose that $G_{1}$ is a pre-compact open subset of $G$ such that $G-G_{1}$ has no compact components. Let $K$ be a compact subset of $G_{1}, K_{1}$ a compact subset of $M_{1}$. Then if $\left\{v_{t}\right\}$ is an analytic family of solutions of $A_{t} v_{t}=0$ in $G_{1}$, for prescribed $\epsilon>0$ and integer $k$, there exists an analytic family $\left\{u_{t}\right\}$ of solutions of $A_{t} u_{t}=0$ in $G$ such that $\left|D^{\beta} u_{t}-D^{\beta_{v_{t}}}\right|<\epsilon$ on $K$ for $t \in K_{1},|\beta| \leqq k$.

The conclusions of Theorems 3 and 4 may be strengthened by asserting that we can approximate a given fundamental solution or solution for fixed $t$ in any prescribed neighborhood in $C^{\infty}(G)$.

Let us conclude with the remark that the methods used in the proof of the above results of $\S \S 1$ and 2 are a development of the arguments given by the writer in [1] and, unlike the arguments of Malgrange in [6], make no use of the theory of topological tensor products.

\section{BIBLIOGRAPHY}

1. F. E. Browder, Functional analysis and partial differential equations. II, Math. Ann. 145 (1962), 81-226. 
2. - Analyticity and partial differential equations. I (to appear).

3. L. Hormander, Operators of principal normal type, Lecture notes, A.M.S. Summer Institute on Functional Analysis, Stanford, Calif., August, 1961.

4. K. Kodaira and D. C. Spencer, On deformations of complex analytic structures. III, Stability theorems for complex structures, Ann. of Math. (2) 71 (1960), 43-76. 1953.

5. J. Leray, Hyperbolic equations, Institute for Advanced Study, Princeton, N. J.,

6. B. Malgrange, Existence et approximation des solutions des equations aux derivees partielles et des equations de convolution, Ann. Inst. Fourier 6 (1956), 271-355.

7. L. Schwartz, Theorie des noyaux, Proc. Internat. Congress Math. (Cambridge, Mass., 1950), Vol. 1, pp. 220-230, Amer. Math. Soc., Providence, R. I., 1952.

Massachusetts Institute of Technology

\title{
SOME CONVOLUTION ALGEBRAS OF MEASURES ON $[1, \infty)$ AND A REPRESENTATION THEOREM FOR LAPLACE-STIELT JES TRANSFORMS
}

\author{
BY LOUIS BRICKMAN
}

Communicated by Einar Hille, April 19, 1962

1. Introduction. In [1] we studied the set $a_{R}$ of power series $\sum_{n=1}^{\infty} a_{n} z^{n}$ convergent for $|z|<R, 0<R \leqq 1$, under the multiplication

$$
\left(\sum_{n=1}^{\infty} a_{n} z^{n}\right)\left(\sum_{n=1}^{\infty} b_{n} z^{n}\right)=\sum_{n=1}^{\infty}\left(\sum_{r=n} a_{r} b_{s}\right) z^{n} .
$$

It was found that $a_{R}$, with the usual addition and scalar multiplication, and with the topology of uniform convergence on compact subsets of the disk $|z|<R$, is a locally convex algebra with identity. Also $\sum_{n=1}^{\infty} a_{n} z^{n}$ is invertible (has an inverse in $a_{R}$ with respect to the above multiplication) if and only if $a_{1} \neq 0$. As a consequence we obtained the following expansion theorem for analytic functions (E. Hille [2]).

THEOREM. Let $f(z)$ be analytic for $|z|<R, 0<R \leqq 1$, with $f(0)=0$. Then associated with any function $g(z)$ analytic in $|z|<R$ with the properties $g(0)=0, g^{\prime}(0) \neq 0$, there is a unique expansion of the form

$$
f(z)=\sum_{n=1}^{\infty} c_{n} g\left(z^{n}\right), \quad|z|<R .
$$

Our object in this paper is to obtain an analogous result for LaplaceStieltjes integrals (Theorem 1 below). We shall base the discussion on the theory of convolution algebras of complex measures on $[0, \infty)$ 